\title{
Limits on the Multi-TeV Cosmic Ray Electron Flux from CREST (Cosmic Ray Electron Synchrotron Telescope)
}

\author{
James Musser* \\ Indiana University, Bloomington, IN 47405 USA \\ E-mail: jmusser@indiana.edu \\ C. Bower ${ }^{1}$, S. Coutu ${ }^{2}$, J. Gennaro ${ }^{3}$, M. Geske ${ }^{2}$, D. Müller ${ }^{4}$, S. Nutter ${ }^{5}$, N. Park ${ }^{4}$, M. \\ Schubnell ${ }^{3}$, G. Tarlé ${ }^{3}$, S. Wakely ${ }^{4}$ \\ ${ }^{1}$ Indiana University, Bloomington, IN 47405 USA \\ ${ }^{2}$ Pennsylvania State University, University Park, PA 16802 USA \\ ${ }^{3}$ University of Michigan, Ann Arbor, MI 48109 USA \\ ${ }^{4}$ University of Chicago, Chicago IL 60637 USA \\ ${ }^{5}$ Northern Kentucky University, Highland Heights, KY 41099 USA
}

\begin{abstract}
The CREST instrument is designed to detect cosmic electrons at energy above $2 \mathrm{TeV}$, which would likely originate in the local Galactic neighborhood owing to their rapid energy loss rates during propagation. CREST detects electrons through their geo-synchrotron x-ray emission, which at these energies is emitted along the direction of electron travel, resulting in a co-linear, isochronous arrangement of $\mathrm{x}$-ray hits in the detector. To observe this signature, an array of $1024 \mathrm{BaF}_{2}$ crystal detectors was built, surrounded by veto plastic scintillators to guard against chance alignments of charged particles in air showers. This instrument was flown on a high-altitude balloon for 10 days in Antarctica during the 2011/2012 season. In this paper the analysis of this data is described, a limit is placed on the number of electrons with energies above $15 \mathrm{TeV}$ observed during the flight, and the principal backgrounds to their signature are characterized.
\end{abstract}

The 34th International Cosmic Ray Conference,

30 July- 6 August, 2015

The Hague, The Netherlands

\footnotetext{
* Speaker.
} 


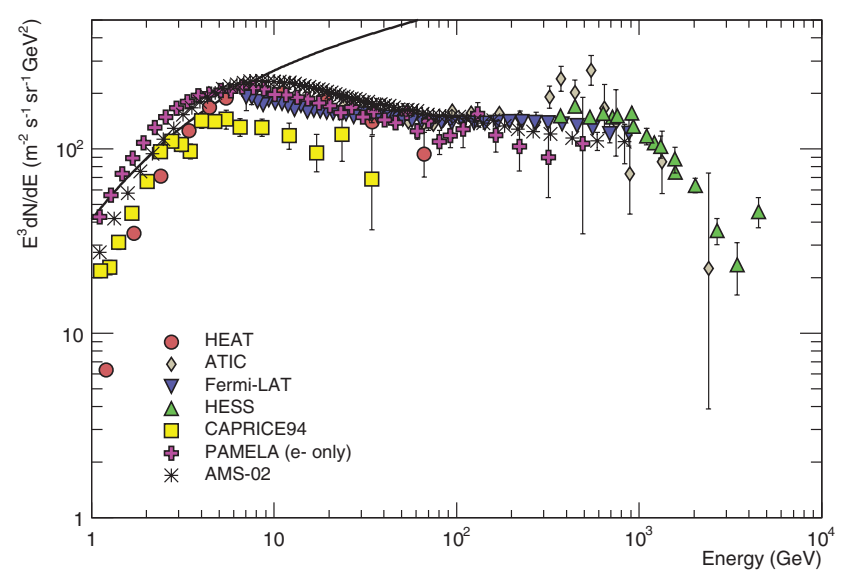

Figure 1: All-electron energy spectrum measurements to date. The black line represents the proton spectrum scaled by 0.01

\section{Introduction}

Electrons of energy above a few $\mathrm{TeV}$ are a largely unexplored component of the cosmic-ray flux at the Earth despite strong evidence of their presence in a number of supernova remnants (e.g., SN 1006). The detection of such electrons at Earth would be extremely significant, yielding information about the spatial distribution of nearby cosmic-ray sources. Electromagnetic energy losses during propagation are so severe that a $1 \mathrm{TeV}$ electron observed at the Earth is likely to have originated within a distance of less than $1 \mathrm{kpc}$. If high-energy cosmic-ray electrons originate in supernova shock acceleration processes, as is the current paradigm for cosmic rays in general [1], just a few supernova remnants are known within this horizon from which these particles could originate. The spectral shape of high-energy electrons should, therefore, be strongly affected by the number of nearby sources, and their space-time distribution [2].

The current status of experimental measurmeents of the high-energy electron flux as a function of energy is shown in Figure 1, which makes clear that even the most recent large acceptance/long exposure instruments have not provided measurements above a few TeV. To extend the energy reach of electron flux measurements, we adopted a novel approach to the detection of electrons of energies between 5 and $50 \mathrm{TeV}$, using a technique for the identification of high-energy electrons proposed years ago [3] [4], but never implemented. We describe this technique and the instrument developed to utilize it next.

\section{Detector Overview}

Detection of primary electrons with energies greater than $5 \mathrm{TeV}$ is achieved in the CREST detector through the observation of the electrons' geo-synchrotron radiation in the hard x-ray region, resulting in large effective detector apertures since the instrument need only intersect the line of synchrotron photons and not necessarily the trajectory of the electron itself. To separate synchrotron-induced events from background photons, two characteristics of the radiation are exploited - the formation of a line of photons at the detector and the very short time interval over 


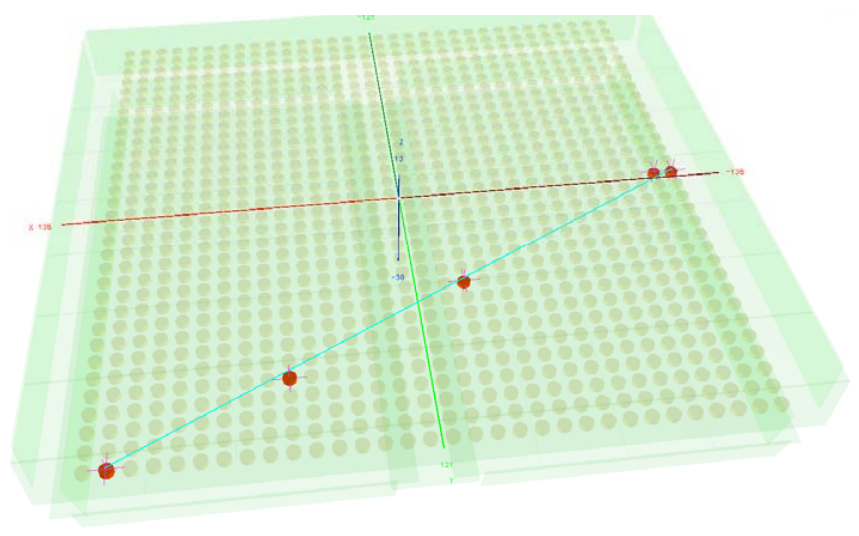

Figure 2: Event display of a signal-like event from the flight in Antarctica.

which these photons are deposited. This is accomplished in the CREST instrument by using an array of 1024 Barium Fluoride $\left(\mathrm{BaF}_{2}\right)$ detectors, each with a diameter of $5 \mathrm{~cm}$ and a thickness of 2 $\mathrm{cm}$. These detectors provide a single hit timing resolution of roughly $1 \mathrm{~ns}$, and an energy resolution of roughly $12 \%$ at $511 \mathrm{keV}$. The crystal array is surrounded on all sides by a $0.5 \mathrm{~cm}$ thick plastic scintillator veto shield which provides rejection against the copious flux of charged particles. Figure 2 is a display of a flight event, showing the layout of the detector, and an event candidate which exhibits the signture of an electron.

The event readout trigger is based on the number of $\mathrm{BaF}_{2}$ hits occurring within a 100 ns time window. For triggering purposes, the $\mathrm{BaF}_{2}$ array is segmented into blocks of $4 \times 4$ crystals. If three or more of these blocks contain hits occurring within a trigger time window, an event readout occurs. In addition, a pre-scaled minimum bias trigger is used, such that every $450^{\text {th }}$ event with any level of crystal activity is read out.

\section{Flight Overview}

The long-duration balloon (LDB) flight of the CREST instrument took place during the 2011/12 Antarctic season, with launch on December 25, 2011, and termination on January 5, 2012. Figure 3 shows the atmospheric pressure measured at the instrument as a function of time during the flight. This is a particularly important parameter for CREST, as the effective area of the instrument is a decreasing function of the atmospheric overburden due to the absorption of synchrotron photons, while the background rates increase with overburden. As this figure demonstrates, the desired 4 $\mathrm{g} / \mathrm{cm}^{2}$ atmospheric overburden depth was not achieved during the flight.

At roughly 3 days into the flight, the instrument began experiencing rate-related data acquisition failures that necessitated the removal of half the $\mathrm{BaF}_{2}$ detectors from acquisition. For the subsquent 3.5 days, 16 of the full 32 columns of $\mathrm{BaF}_{2}$ detectors were read out. For the remainder of the flight, the instrument was operated in a checkerboard configuration of $4 \times 4$ blocks of active $\mathrm{BaF}_{2}$ detectors. These configurations were individually modelled in the detector simulations used in the analysis described below. The average live time fraction achieved during flight was $60 \%$, with the majority of the dead time resulting from detector tuning and calibration. The re- 
sults described in the next sections are based on data taken in the half detector and checkerboard configuration, with work on the full detector data set nearing completion.

\section{Data Analysis}

The first stage of data analysis consisted of developing and applying calibrations which convert the raw $\mathrm{ADC}$ and TDC data from the $\mathrm{BaF}_{2}$ and veto detectors into hit times and detector energy depositions, and the removal of time or temperature dependence in these conversions. The detector performance achieved in these calibrated quantities is fully consistent with the $1 \mathrm{~ns}$ hit time resolution and $12 \%$ energy resolution at $511 \mathrm{keV}$ expected from bench measurements.

In the CREST data set we are searching for rare electron-induced events in a sea of potential backgrounds, and therefore the modelling of these backgrounds is of primary importance. For this purpose, we adopt the GLAST/Fermi atmospheric $\gamma$ ray and particle flux model, which includes both upward and downward going components [5]. Systematic errors in the background particle flux estimates were assessed based on a comparison between results using this model, and the QARM model [6]. All critical aspects of the flight conditions were simulated, including the variation of depth with time, changes to detector configuration, etc. A significant component of the analysis effort was verifying that the instrument model accurately describes the flight data. The plot in Figure 4, showing a comparison between the reconstructed event spatial extent in the $\mathrm{BaF}_{2}$ array along its principal axis for flight data, shown in black, simulated background events shown in red, and for simulated electron events in blue, is representative of the excellent agreement between data and Monte Carlo.

The instrumental signature of a 'golden'synchrotron event from a high-energy electron is a set of co-linear, isochronous crystal hits with no activity in the veto system. The analysis of crystal event data begins with a clustering algorithm that seeks to combine neighboring crystal hits resulting from a single incident gamma ray. The spatial and temporal locations of these clusters are then used in a search for linear, isochronous signal events. Additional requirements are placed on the

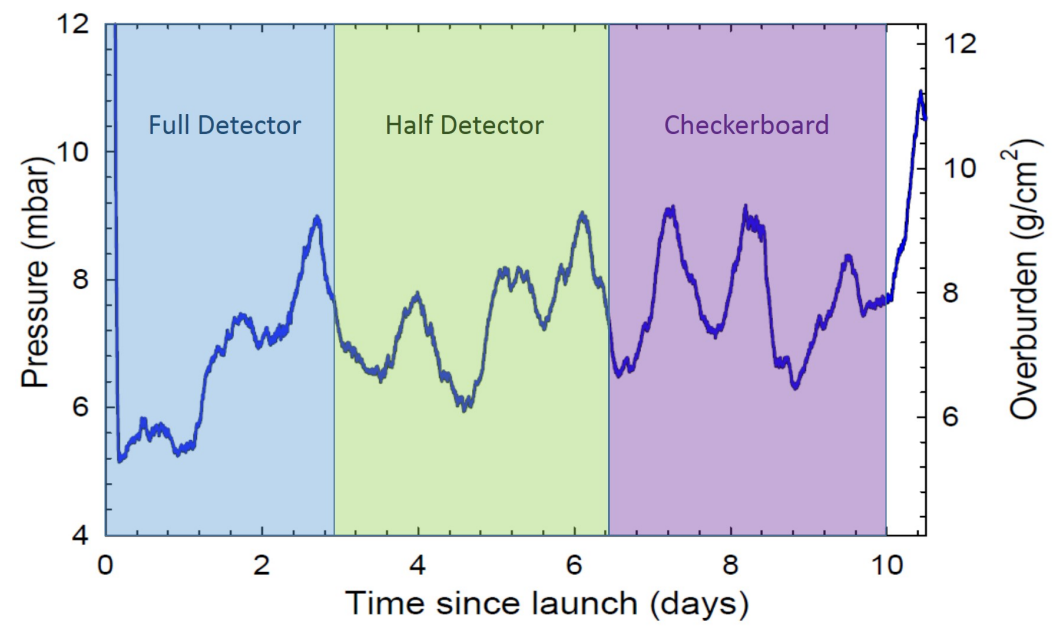

Figure 3: Flight pressure profile. Also shown are the time periods corresponding to the three detector configurations used. 


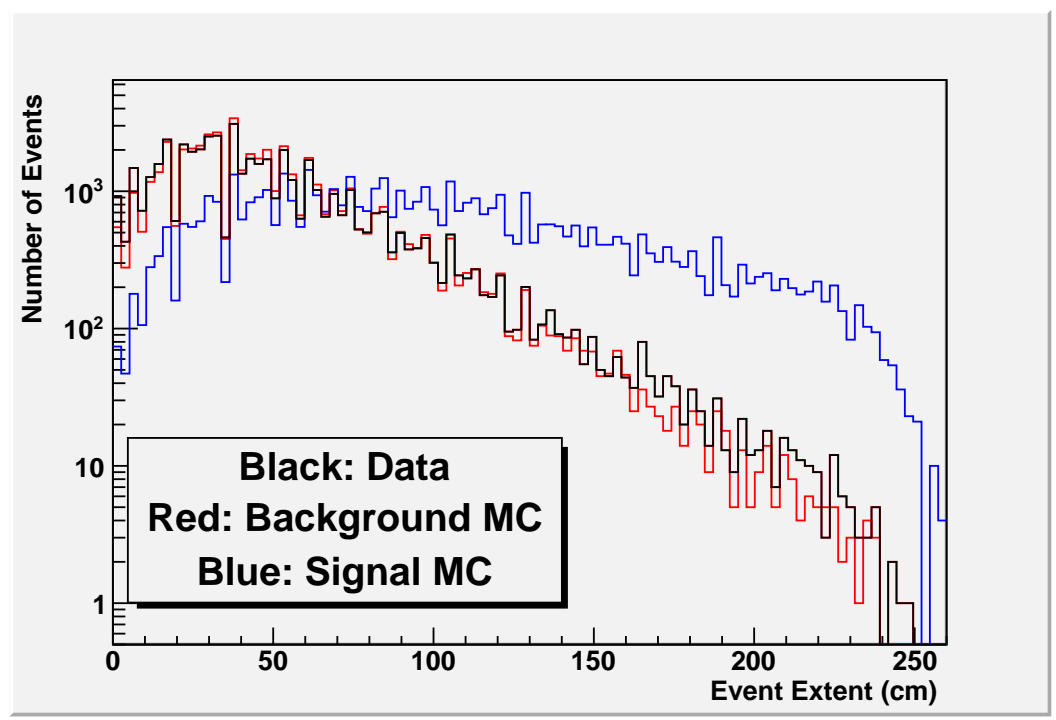

Figure 4: Event spatial extent along the principal axis, in $\mathrm{cm}$.

minimum extent of the event along the event axis, and the presence of gaps between clusters, to further suppress the background resulting from charged particles producing a linear track in the crystal array. In Table 1 we summarize the data selections used, and compare the event rates for the half detector configuration after each selection is applied in data and Monte Carlo. Table 1 also contains the selection efficiency for $20 \mathrm{TeV}$ electrons associated with each data selection. The electron selection efficiency is a relatively weak function of electron energy at energies above 20 $\mathrm{TeV}$. At lower energies, the selection efficiency associated with the requirement that no veto system activity is observed decreases significantly, resulting in an effective threshold for signal events of approximately $15 \mathrm{TeV}$, somewhat above the $5 \mathrm{TeV}$ intrinsic threshold for the synchrotron technique. This results from the increasing probability at low energies that the synchrotron $\mathrm{x}$-rays responsible for the event trigger are accompanied by the primary electron itself in the detector acceptance. An important point to note is that the low overall value for the electron selection efficiency shown in Table 1 is largely a consequence of accepting at the trigger level events which do not satisfy the ncluster $>3$ requirement.

Table 1: Event Selection Summary

\begin{tabular}{|c|c|c|c|}
\hline \hline Event Selection & Rate $\mathrm{Hz}($ Data) & Rate $\mathrm{Hz}(\mathrm{MC})$ & 20 TeV Electron Selection Eff. \\
\hline raw (hardware trigger rate) & 3055 & 3177 & 1.0 \\
no veto activity & 277 & 181 & 0.14 \\
\# clusters $>3$ & 8.4 & 7.4 & 0.056 \\
event extent $>75 \mathrm{~cm}$ & 3.2 & 2.7 & 0.86 \\
largest inter-cluster gap $>40 \mathrm{~cm}$ & 2.2 & 1.4 & 0.99 \\
hit time vs position $\chi^{2}<5$ & 1.9 & 1.3 & 0.98 \\
crystal x vs y fit $\chi^{2}<5$ & 0.064 & 0.04 & 0.30 \\
\hline
\end{tabular}

In Figure 5 we present the $1 / v$ distribution of events in the flight data for the half-detector 
configuration, and which satisfy all the criteria summarized in Table 1. Here, $v$ is the propagation velocity of the event in the crystal array along its major axis. The peak seen in this figure corresponds to the propagation of hit times in the crystal array at a velocity $v=c$. This is characteristic of background events arising from interactions in the detector, or from a charged particle which fails to produce signals in the veto detectors. The signal produced by synchrotron-induced events does not exhibit this behavior, as the synchrotron photons propagate on a common wave front that is perpendicular to the local magnetic field direction. In the case of a near-vertical magnetic field, as in a southern LDB flight, the synchrotron photons arrive at the detector at essentially the same instant, independent of the angle of incidence of the primary electron. Such a signal would be seen as an excess in events near $1 / v=0$, as shown in Figure 5. A limit on the rate of signal events passing event selections is obtained by fitting the data $1 / v$ distribution to scaled Monte Carlo background and signal distributions, and dividing the integral of the scaled signal distribution by the corresponding data live time. Through this procedure we obtain a $90 \%$ upper limit on the rate of signal events of $R_{\text {cand }}<5.4 \times 10^{-4} \mathrm{ev} / \mathrm{s}$. Unlike most traditional detectors, the effective acceptance of the CREST instrument is a roughly linearly increasing function of the primary electron energy, as shown in Figure 6. This is a consequence of the increase in synchrotron photon production rates and energies with increasing primary energy. Therefore, an electron energy distribution must be assumed in obtaining an integral flux limit based on the signal event rate limit above. For an $E^{-3}$ electron energy distribution, an integral flux limit above the selection efficiency threshold of $15 \mathrm{TeV}$ of $f<7.11 \times 10^{-3} \mathrm{~m}^{-2} s r^{-1} \mathrm{~s}^{-1}$ is obtained at the $90 \%$ confidence level. A more steeply falling electron spectrum (or as is most likely, a spectral cut off) would result in a somewhat higher flux limit as a result of the energy-dependent effective acceptance of the instrument. For example, the flux limit for an assumed $E^{-4}$ spectrum is roughly $4 \%$ higher than the value quoted above. This limit is based only on topological information, and does not exploit additional background rejection power stemming from the requirement that the pulse height information in the $\mathrm{BaF}_{2}$ array be consistent with synchroton x-rays from an electron with energy greater then $15 \mathrm{TeV}$. Work on finalizing an energy-based event selection is nearing completion, and will be included in the final publication of this work. Studies to date indicate that energy-based event selections will improve background rejection by roughly $20 \%$.

\section{Characterization of Backgrounds}

The characterization of the background events which survive the data selections above is useful in evaluating the limits of this electron detector technique. We summarize these results in Table 2. They are relatively insensitive to the detector configuration, and represent an average over the depth profile for the flight. The dominant background, representing 55\% of the full background rate, is due to atmospheric gammas interacting in the instrument and producing a random coincidence of co-linear, isochronous hits in the $\mathrm{BaF}_{2}$ array. Upward going secondary gammas are responsible for more than half of these background events. One should note that this background component would also be present at similar levels in a space-based measurement using this technique. It is also worth noting that one quarter of the background events in our final event sample originate from primary protons which the veto system failed to reject. Even though extraordinary measures were taken in the detector design to maximize the hermeticity (over $99 \%$ coverage was achieved) 


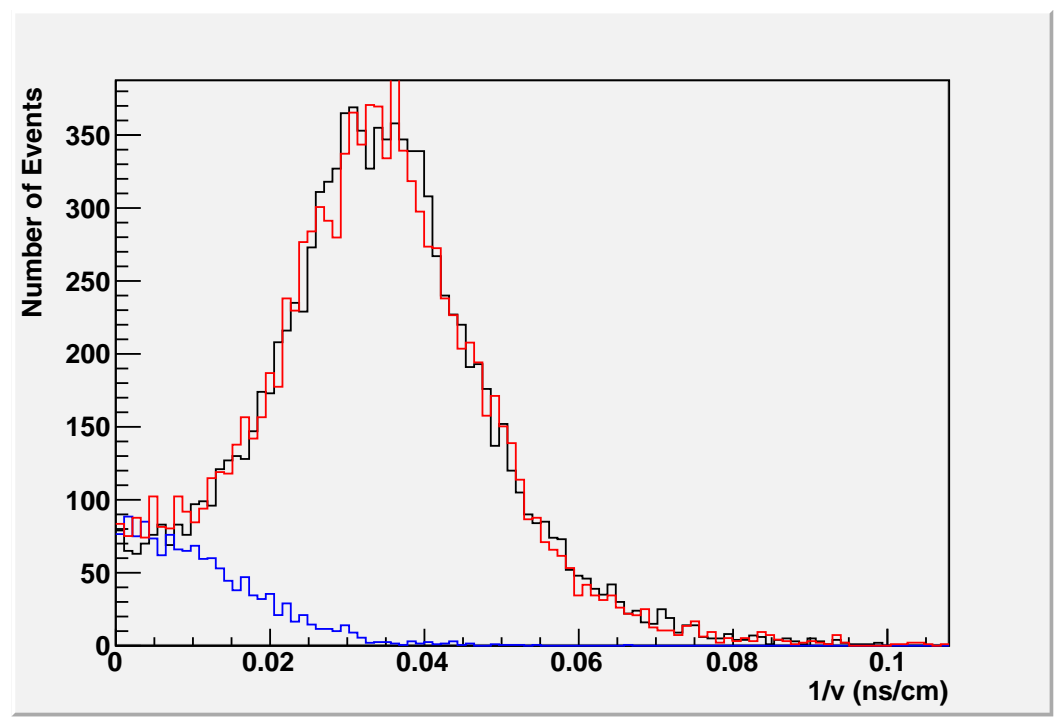

Figure 5: Inverse event propagation speed in the $\mathrm{BaF}_{2}$ array. Black: Flight data passing selections in Table 1 for the half-detector configuration. Red: Monte Carlo background prediction. Blue: Synchrotron event prediction (arbitrary scale).
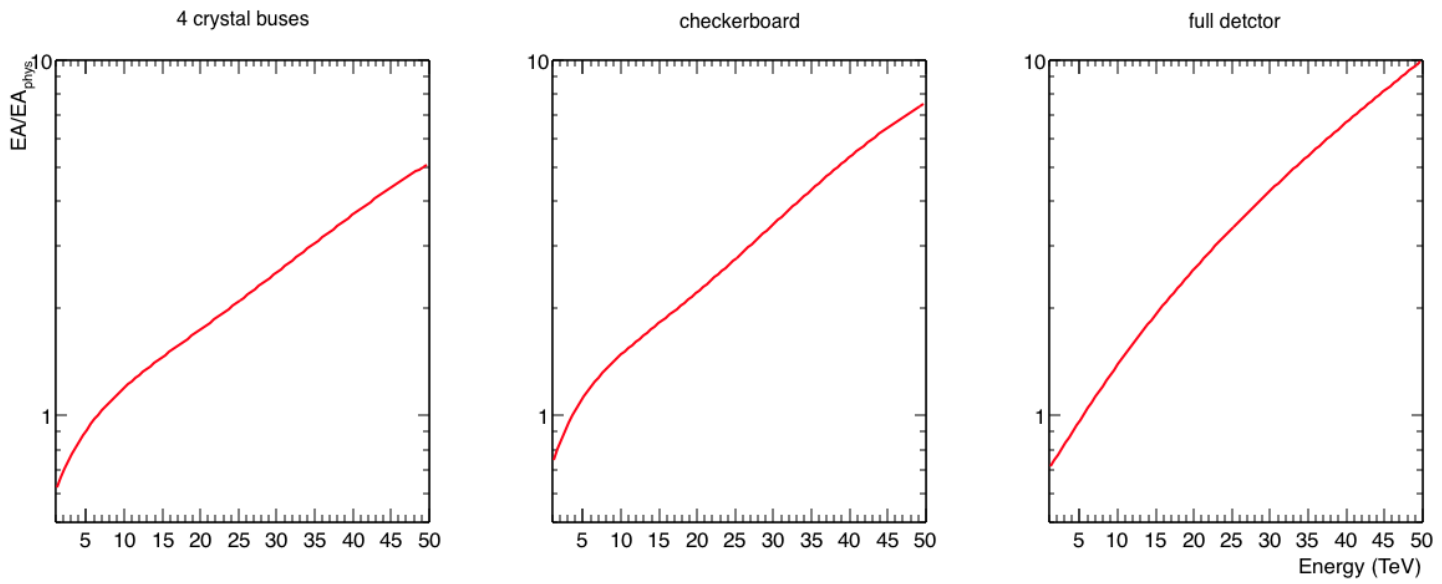

Figure 6: The effective acceptance of the CREST instrument as a function of electron energy, for the three detector configurations. The effective acceptance is shown in units of the physical acceptance of the instrument, which is $18.1 \mathrm{~m}^{2} \mathrm{sr}$.

and efficiency of the veto system, charged particle-initiated events remain a major contributor to background rates. The arrangement of the $\mathrm{BaF}_{2}$ crystals in a flat array increases the probability that a charged particle will produce a pattern of detector hits characteristic of signal events. It is likely that an alternative, non-coplanar array layout would substantially reduce this background.

\section{Conclusion}

In this paper, we have presented preliminary results for a limit on the integral flux of UHE electrons for energies greater than $15 \mathrm{TeV}$ based on data from the CREST LDB flight of 2011/12. 
Table 2: Background event type fraction

\begin{tabular}{|c|c|}
\hline \hline Background event type & Fraction of total rate after event selection \\
\hline Upward secondary gammas & 0.3 \\
Downward secondary gamma & 0.25 \\
Primary charged particle & 0.25 \\
Neutrons & 0.07 \\
Low-energy electron & 0.055 \\
Secondary charged particles & 0.045 \\
Primary gamma & 0.03 \\
\hline
\end{tabular}

The CREST experiment represents the first attempt at utilizing the synchrotron radiation technique for measuring the UHE electron flux, and illustrates some of the challenges faced in a practical implementation of the technique. Among these are event selection inefficiencies resulting from activity other than that produced by the synchrotron photons (for example, from the UHE electron itself) complicating the event topology in the $\mathrm{BaF}_{2}$ array, and producing activity in the veto system. In a sub-orbital experiment such as CREST, backgrounds due to secondary production in the atmospheric overburden are significantly increased, and the production of high-energy bremsstrahlung in the atmosphere and its subsequent interaction in the detector significantly decreases event selection efficiencies. A space-based version of this experiment would avoid many of these issues, although the background from secondary albedo gamma rays is likely to remain a challenge. A second challenge facing future space-based implementations of this technique will be the design of a veto system with the hermeticity needed to reduce to manageable levels the extremely copious backgrounds from charged particles mimicking the synchrotron event signature, while minimizing the veto system's rejection of true UHE electron events.

Acknowledgements: This work has been supported under NASA Grants NNX08AC42G, NNX08AC40G, NNX08AD08G, and NNG04WC34G, and the Kentucky Space Grant. We would like to thank the Columbia Scientific Balloon Facility and the CSBF launch crews for their excellent support of balloon missions. The collaboration also gratefully acknowledges the contributions of T. Anderson, J. Ameel, J. Bench, T. Bishay, B. Dingee, M. Gebhard, M. Grinshpon, G. Kelderhouse, B. Kunkler, B. Martin, R. Northrop, A. Shroyer, C. Smith, and K. Wallace.

\section{References}

[1] M. Ackermann et al., Science 339,807 (2013).

[2] T. Kobayashi et al., ApJ 601, 340 (2004).

[3] O.F. Prilutskiy, ZhETF 19, 320 (1972).

[4] S.A. Stephens \& V.K. Balasubrahmanyan, J. Geophys. Res. 88, A10 (1983).

[5] T. Mizuno et al., ApJ 614,1113 (2004).

[6] F. Lei al. IEEE Transaction on Nuc. Sci., 51, 6 (2004). 